\section{Unmoderated Posters: Technology and Instruments}

\author{
UP-66 \\ A Comparison of Prone versus Lateral Decubitus Positioning in \\ Percutaneous Nephrostomy Tube Insertion \\ Wu, Christopher; Kwong, Justin; Lindsay, Leanne; Vora, Parag; Davies, \\ Timothy \\ McMaster University, Hamilton, ON, Canada \\ Introduction and Objectives: No consensus exists on the best position for \\ percutaneous nephrostomy ( $\mathrm{PCN}$ ) tube insertion, though prone position- \\ ing is the most widely used, thought to minimize intraperitoneal injuries. \\ However, there is a risk of hemodynamic compromise with this approach \\ with turning the patient from supine to prone position. We describe a new \\ technique of ultrasound guided PCN access in lateral decubitus position \\ under local anesthetic. Our objective was to compare the complications \\ and morbidities after the insertion of a PCN tube in prone or lateral \\ decubitus position. \\ Methods: Retrospective data collection was carried out on all adult \\ patients that underwent PCN insertion at our institution from August \\ 2008-August 2012. A total of 336 patients (161 men and 175 women) \\ underwent PCN. With regards to positioning, 237 (70.5\%) were inserted \\ prone and $99(29.5 \%)$ were inserted in lateral decubitus position. \\ Results: Univariate analysis between the two techniques showed no sig- \\ nificant difference in 30-day complications requiring replacement $(10.1 \%$ \\ prone, $14.1 \%$ lateral; $p=0.289)$, post-procedural ICU admission $(5.8 \%$ \\ prone, $5.1 \%$ lateral; $\mathrm{p}=0.757)$, post-procedural transfusion $(0.4 \%$ prone, \\ $0 \%$ lateral; $p=0.517$ ) or post procedural hospitalization over 48 hours \\ ( $2.5 \%$ prone, $2.0 \%$ lateral; $p=0.779)$. A significant increase in 30 -day \\ mortality was seen in the lateral decubitus group $(11.1 \%$ lateral, $3.4 \%$ \\ prone; $\mathrm{p}=0.005$ ), however, on further review these patients died as a \\ result of underlying pathology and were under palliative measures prior \\ to the procedure. \\ Conclusions: Our data show that the complications and morbidities asso- \\ ciated with both prone and lateral approaches to percutaneous neph- \\ rostomy tube insertion were not significantly different, suggesting that \\ lateral decubitus positioning is a safe and effective alternative to prone \\ positioning. Review of current literature shows no prior research describ- \\ ing routine lateral decubitus PCN tube placement.
}

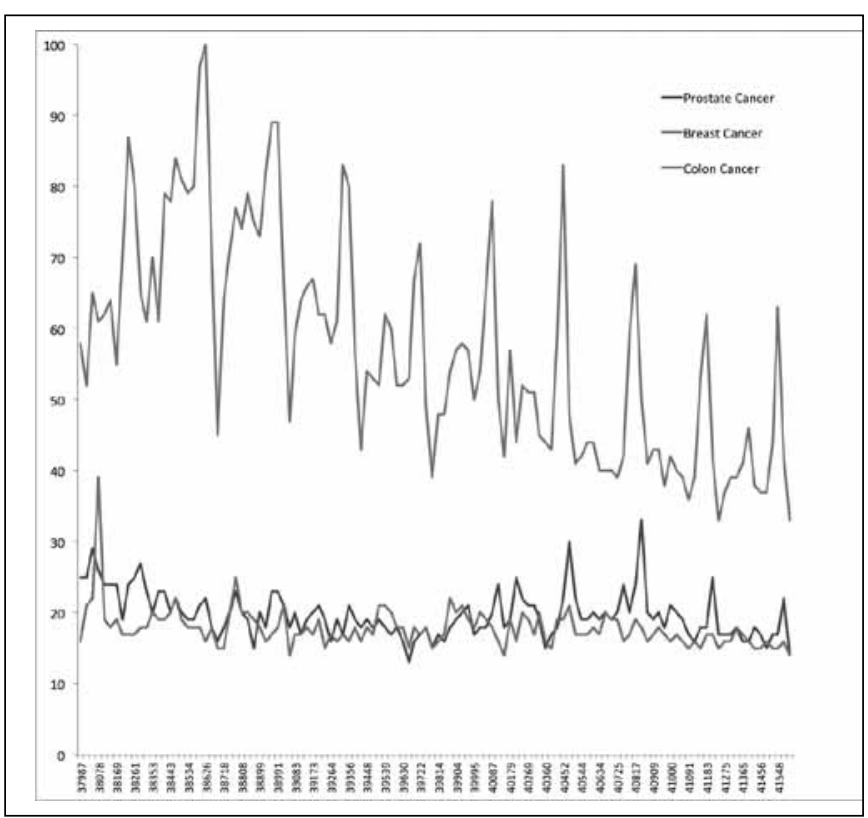

Fig. 2. UP-65. Relative search volumes in Canada for the terms "prostate cancer", "breast cancer" and "colon cancer" from 2004-2013. Data are normalized to reflect the proportion of searches for a given term relative to the others and to the total volume of Google searches for all terms at a given time point. 